\title{
EDITORIAL
}

\section{“Dysautonomia": a plea for precision}

\author{
Eduardo E. Benarroch ${ }^{1}$
}

Received: 16 November 2020 / Accepted: 17 November 2020 / Published online: 2 January 2021

(c) Springer-Verlag GmbH Germany, part of Springer Nature 2021

Keywords Postural tachycardia syndrome $\cdot$ POTS $\cdot$ Orthostatic intolerance $\cdot$ Syncope $\cdot$ Autonomic dysfunction

The current state of development of medical knowledge, based in part on next generation sequencing and other advanced genomic approaches and the identification of signals involved in innate and adaptive immunity, has made imperative for the clinician to define disease phenotypes as accurately as possible. This is necessary both for appropriate interpretation of the increasing information provided by basic research and for its application to the evaluation and management of patients in an individualized manner. Physicians managing patients referred for autonomic disorders face unique challenges compared to those practicing other subspecialties. I will mention four challenges that, in my view, must be urgently considered and addressed.

The first challenge is the definition of "dysautonomia." In his superb Companion to Clinical Neurology, William Pryse-Phillips defines dysautonomia (dys-, "bad", and autonomia- Greek for "freedom to use its own laws") as "any disorder of the autonomic nervous system, sympathetic or parasympathetic, including hyper- as well as hypofunction". In Dorland's Illustrated Medical Dictionary, "dysautonomia" is defined based on the features of familial dysautonomia (Riley-Day syndrome, or hereditary sensory and autonomic type 3), namely impaired visceral afferent function [1]. In Pryse-Phillips's book, "weakness" is defined as "a decrease in the muscular activity exerted in the performance of a resisted task as judged by the patient or the examiner". Of course, no neurologist would be satisfied using "weakness" as a patient's final diagnosis. On the contrary, a neurologist must determine if the weakness is due to involvement of corticospinal tract, lower motor neuron, neuromuscular junction, or muscle. Unfortunately, this is not the same with autonomic disorders. Many physicians

Eduardo E. Benarroch

Benarroch.eduardo@mayo.edu

1 Department of Neurology, Mayo Clinic, 200 1st St SW, Rochester, MN 55905, USA believe that "dysautonomia" is a final diagnosis rather than an imprecise description. Worse still, many physicians and patients wrongly equal postural tachycardia syndrome (POTS) with "dysautonomia", and use both terms interchangeably. Indeed, some advocacy groups of patients with POTS have unabashedly hijacked the term dysautonomia. In reality, there is overwhelming scientific evidence proving that, rather than a disorder of the autonomic nervous system, POTS is a highly heterogeneous syndrome with multiple comorbidities and different possible pathophysiological mechanisms [2]. Moreover, the magnitude of the heart rate increase when standing - the paradigmatic autonomic marker of this syndrome-is unrelated to the symptomatic burden of patients with POTS [5]. Symptoms interpreted as "autonomic" may reflect abnormal failure or hyperactivity of the sympathetic or parasympathetic output; a physiological response to a physical, homeostatic, or psychological challenge; or interoceptive input originating from dysfunctional visceral organs or effector (more about this in the third challenge), including the skin-as in the case of thermal discomfort. Thus, whereas a Babinski sign, slow axon conduction velocities, or electromyography motor unit potential abnormalities are almost always pathological, a delayed tilt-induced hypotension after nitroglycerine challenge, or a noteworthy increase in heart rate when standing are not necessarily so. Constipation and urinary retention due to poor pelvic floor relaxation, relatively frequent in the general population, are not manifestations of an autonomic disorder. Thus precision in the definition leads to a reasonable pathophysiological hypothesis and differential diagnosis, appropriate testing, and, most importantly, adequate therapy.

The second challenge is the confounding factors surrounding autonomic testing. The autonomic laboratory evaluation is an extension of the clinical evaluation. An extension, not a substitution. In my experience, few physician "specializing" in POTS or other disorders mislabeled as "dysautonomia" perform an appropriate history and clinical 
examination, including bedside supine and active standing measurements of blood pressure and heart rate, assessment of skin color or moisture, and size, symmetry and reactivity of pupils [8]. Unlike the case of the EMG, where there is a direct recording of the response of a sensory or motor nerve to electrical stimulation, clinical autonomic testing does not record the activity of sympathetic or parasympathetic nerves. It infers the normality or abnormality of sympathetic or parasympathetic pathways based on the response of sweat glands, sinus node, or resistance blood vessels in response to physiological stimuli. Unlike the EMG, where the magnitude of the electrical stimulus is less relevant than the maximum obtained response, the magnitude and quality of the stimulus for autonomic testing is critical. Thus, skin resistance affects the latency of a sweat response to iontophoresed acetylcholine in quantitative sudomotor axon reflex tests (QSART); and respiratory effort affects the magnitude of the heart rate and blood pressure responses during deep breathing and the Valsalva maneuver. A third and fundamental difference between EMG and autonomic studies is the effect of drugs on the magnitude of the recorded responses. Whereas the typical "confounding" drug in EMG is an inhibitor of the acetylcholinesterase, the list of drugs that can affect the response of autonomic effectors is sizable and expanding. Some drugs with "expected" anticholinergic effects such as amitriptyline, doxepin, or diphenylamine, or with "expected" effects on blood pressure such as adrenergic or calcium channel blockers, diuretics, or angiotensin system inhibitors are obvious candidates that are typically asked to be discontinued at least 2 days prior to testing (which is ideal but unfortunately not necessarily attained in all patients). Other commonly use drugs, including topiramate, bupropion, mirtazapine, and clozapine can substantially impair the effector responses during autonomic testing, yet there are not frequently considered potential confounders by many physicians. Needless to mention the effect of stimulants (e.g., amphetamine, methylphenidate, atomoxetine) on heart rate. Thus, physician education on basic autonomic pharmacology is critical. Drug discontinuation for 3-4 half-lives prior to testing is typically thought to be enough to restore baseline receptor activity. However, to my knowledge, whether the same applies after long-term use promoting pre- or postjunctional receptor up- or down-regulation has not been appropriately addressed.

A third challenge that must be urgently addressed is the pervasive confusion of visceral symptoms with autonomic dysfunction. The wide application of patient-reported questionnaires have led to the concept of "frequent and underrecognized" autonomic symptoms in many neurologic and non-neurologic disorders This applies not only to disorders with established mechanistic basis for autonomic failure, such as Parkinson disease, but also to those where the symptoms are misinterpreted as reflecting autonomic dysfunction when they rather reflect increased awareness of visceral sensation, chronic illness associated with inflammation, deconditiong, or combinations thereof. Afferent mechanisms trigger symptoms that are misinterpreted as "dysautonomia." A typical example is pelvic floor dysfunction. This may lead to constipation due to dyssynergic defecation [14] or to voiding dysfunction, bladder distension and urinary retention [7, 12]. Excessive pudendal nerve afferent activity from the pelvic floor or inputs from the distended rectum may inhibit detrusor contraction and thus the micturition reflex [15]. These disorders, in the strict sense, are nor primarily due to autonomic nervous system dysfunction. According to the predictive coding model, the brain interprets the dynamic changes of viscerosensory information by predicting the likely causes of those changes $[1,13]$; prior interoceptive experiences create predictions that are sent to effector organs as probable interoceptive consequences of the effect of autonomic output to the target. The difference between the expected and the actual afferent interoceptive information generates a prediction error signal that triggers an efferent neural drive to the peripheral nervous system to change the internal bodily state and thus minimize prediction errors. Thus, visceral symptoms do not equate to autonomic dysfunction and may reflect complex information processing in the CNS.

A fourth challenge is the misunderstanding of the complex, reciprocal and inextricable neuro-immune interactions. Afferent inputs, autonomic outputs, and signals from immune cells interact at barrier tissues such as the skin and mucosal surfaces (e.g., in the gut and lungs) to maintain tissue homeostasis and coordinate host defense in the face of a variety of environmental threats, including thermal changes, mechanical injury, and microbial pathogens [4]. These interactions are complex, reciprocal, involve different signals, and are still incompletely defined in vivo. Signals from immune cells trigger pain [6]; those from autonomic efferents modulate immune-inflammatory responses [9]; and visceroceptor, nociceptor, and thermoreceptor inputs coordinately modulate autonomic output and inflammation both locally, and via "immune-neuro-immune reflexes" integrated at the level of the brainstem and hypothalamus $[3,10]$. A prominent example is that of "gut-brain interactions", involving the microbiota, enteric immune cells, vagal afferents, and sympathetic efferents to immune organs. Pain and autonomic dysfunction may be salient manifestations of some inflammatory and immune disorders, including autoimmune (including paraneoplastic) ganglionopathies, and contactin-associated protein-like 2 (Caspr2) antibody disorders [11]. Clearly defined immune mechanisms in patients with high titers of autoantibodies provide a strong rationale for immunomodulatory therapy. However, this does not necessarily apply to patients with symptoms suggestive of autonomic impairment and low levels of circulating autoantibodies. In these patients, the lack of objective and reproducible 
autonomic deficits, and the coexistence of alternative explanations for visceral dysfunction or sensitivity strongly argue against the diagnosis of "autoimmune dysautonomia", and provide no clear rationale for immunomodulatory therapy. The anecdotal reports of patients "feeling better" after intravenous immunoglobulins or corticosteroids are not enough to warrant therapy without objective measurements of improved autonomic function (assuming, of course, that those patients had objective autonomic dysfunction to begin with). The same may apply to a sizable number of patients diagnosed with "small fiber neuropathy" based on insubstantial findings.

The specialty of autonomic disorders is challenging, evolving, and relatively young. It requires at least a basic understanding of the anatomy, physiology and pharmacology. This basic knowledge is fundamental for the appropriate interpretation of symptoms, diagnosis, and appropriate therapy. A precise phenotypic characterization is necessary to take advantage of genetic information and immunologic approaches for rational pathophysiological interpretation and management of autonomic disorders. Whereas entering terms such as "recessive ataxia" into available genetic databases may yield useful molecular insights and testable hypothesis for potential-targeted therapies, entering the term "dysautonomia" without a more precise phenotypic characterization will not yield any useful information. It is time to judiciously limit—perhaps even terminate-the use of "dysautonomia", an imprecise, misleading, and hindering term.

Funding None.

\section{Compliance with ethical standards}

Conflict of interests None.

\section{References}

1. Barrett LF, Simmons WK (2015) Interoceptive predictions in the brain. Nat Rev Neurosci 16:419-429

2. Benarroch EE (2012) Postural tachycardia syndrome: a heterogeneous and multifactorial disorder. Mayo Clin Proc 87:1214-1225

3. Benarroch EE (2019) Autonomic nervous system and neuroimmune interactions: New insights and clinical implications. Neurology 92:377-385

4. Blake KJ, Jiang XR, Chiu IM (2019) Neuronal regulation of immunity in the skin and lungs. Trends Neurosci 42:537-551

5. Boris JR, Huang J, Bernadzikowski T (2020) Orthostatic heart rate does not predict symptomatic burden in pediatric patients with chronic orthostatic intolerance. Clin Auton Res 30:19-28

6. Chen O, Donnelly CR, Ji RR (2020) Regulation of pain by neuroimmune interactions between macrophages and nociceptor sensory neurons. Curr Opin Neurobiol 62:17-25

7. Chermansky CJ, Moalli PA (2016) Role of pelvic floor in lower urinary tract function. Auton Neurosci Basic Clin 200:43-48

8. Cheshire WP Jr, Goldstein DS (2018) The physical examination as a window into autonomic disorders. Clin Auton Res 28:23-33

9. Godinho-Silva C, Cardoso F, Veiga-Fernandes H (2019) Neuroimmune cell units: a new paradigm in physiology. Annu Rev Immunol 37:19-46

10. Griton M, Konsman JP (2018) Neural pathways involved in infection-induced inflammation: recent insights and clinical implications. Clin Auton Res 28:289-299

11. Norcliffe-Kaufmann L, Slaugenhaupt SA, Kaufmann H (2017) Familial dysautonomia: history, genotype, phenotype and translational research. Prog Neurobiol 152:131-148

12. Osman NI, Chapple CR (2014) Fowler's syndrome-a cause of unexplained urinary retention in young women? Nat Rev Urol 11:87-98

13. Seth AK, Friston KJ (2016) Active interoceptive inference and the emotional brain. Philos Trans R Soc Lond B Biol Sci 371:9

14. Skardoon GR, Khera AJ, Emmanuel AV, Burgell RE (2017) Review article: dyssynergic defaecation and biofeedback therapy in the pathophysiology and management of functional constipation. Aliment Pharmacol Ther 46:410-423

15. Wyndaele M, De Wachter S, De Man J, Minagawa T, Wyndaele JJ, Pelckmans PA, De Winter BY (2013) Mechanisms of pelvic organ crosstalk: 1. Peripheral modulation of bladder inhibition by colorectal distention in rats. J Urol 190:765-771 